\title{
Entrelacs
}

Cinéma et audiovisuel

$6 \mid 2007$

L'Arbre

\section{Les sous-bois du cinéma}

\section{Caroline Renard}

\section{(2) OpenEdition}

Journals

Édition électronique

URL : http://journals.openedition.org/entrelacs/333

DOI : 10.4000/entrelacs.333

ISSN : 2261-5482

Éditeur

Éditions Téraèdre

Édition imprimée

Date de publication : 1 mars 2007

ISBN : 978-2-912868-70-1

ISSN : 1266-7188

\section{Référence électronique}

Caroline Renard, «Les sous-bois du cinéma », Entrelacs [En ligne], 6 | 2007, mis en ligne le 01 août 2012, consulté le 10 décembre 2020. URL : http://journals.openedition.org/entrelacs/333 ; DOI : https://doi.org/10.4000/entrelacs.333

Ce document a été généré automatiquement le 10 décembre 2020.

Tous droits réservés 


\section{Les sous-bois du cinéma}

\section{Caroline Renard}

1 L'histoire du cinéma est riche de représentations d'arbres privés de ramure. Plans rapprochés d'un arbre seul ou plans de sous-bois, il n'est pas rare que le cadre cinématographique ne s'intéresse finalement qu'au tronc. Le cinéma joue du cadre pour se débarrasser des branchages et nous offrir des images qui coupent les troncs à mihauteur et laissent l'ensemble des ramures hors champ. Je ne me pencherai donc ici ni sur les branches légères ni sur les racines, ces deux pôles d'attraction de l'arbre, mais sur le tronc, ce corps central et massif dont la présence peut, selon les circonstances, accroître le réalisme d'une scène ou, tout aussi bien, ouvrir l'espace filmique à une temporalité autre que celle des personnages. Le tronc est à la fois pour le cinéma un élément du décor, une forme plastique et un objet signifiant ${ }^{1}$. En termes d'analyse filmique, l'intérêt plastique et esthétique de l'arbre s'associe à la pluralité de ses significations. Dès lors, comment conjuguer la présence visuelle de l'arbre dans le système filmique et l'interprétation sémantique de cet objet si particulier?

2 Le tronc sera notre point d'appui, notre socle et l'axe à partir duquel ressaisir le mouvement complexe d'interprétation de l'arbre. Sa forme simple, monotone et visuellement austère, nous permet d'évacuer provisoirement les mythologies et les significations symboliques ou allégoriques de l'arbre. Emprunté à la nature pour participer de l'espace diégétique, le tronc est parfois également réinvesti par les arts plastiques, taillé par les sculpteurs, imité par des installations. Sa structure qui condense le paradoxe de la vie immobile de l'arbre, offre au domaine esthétique une matière d'expérimentation des sens et de la pensée. Avec des exemples empruntés à Edward S. Porter (The Great Train Robbery, 1902), Andrei Tarkovski (L'Enfance d'Ivan,1962), mais aussi au plasticien italien Giuseppe Penone et aux œuvres récentes d'Abbas Kiarostami, nous verrons comment chaque apparition de l'arbre convoque nos expériences physiques et esthétiques de la nature.

3 Même si chacun de nous voit dans l'arbre des réalités différentes, le cinéma instaure un cadre, une position, un angle, une organisation visuelle tels, que l'arbre acquiert un caractère et des propriétés filmiques indéniables. L'échelle scalaire, l'angle de prise de vue, la composition du cadre, le mouvement de caméra déterminent une présence 
visuelle qui fait que face à un plan d'arbre, je ne raisonne pas comme un bûcheron ni comme un menuisier. Je peux me permettre d'oublier momentanément les lois de la botanique. Le cadre est l'élément fédérateur à partir duquel une interprétation de l'arbre peut se construire. Les liens que le cadre établit entre l'arbre représenté et le réel, entre la nature et son imitation, témoignent non seulement de la richesse sémantique de l'arbre mais aussi de la structure de notre propre regard. Cadrer les arbres par le tronc renvoie en effet à la question de l'artialisation in visu (Alain Roger) dont le spectateur est l'objet.

Les péripéties que déroule le récit de L'Attaque du train rapide (The Great Train Robbery) de Edward S. Porter sont connues. Après avoir dévalisé le wagon postal et les passagers d'un train, quatre gangsters s'enfuient dans une forêt. Ils descendent de la voie ferrée à travers un sous-bois où les attendent leurs chevaux. Ils s'enfuient au galop, mais sont bientôt rattrapés par un groupe de soldats. Croyant les avoir semés, les gangsters partagent le butin dans une forêt où ils sont retrouvés puis abattus par leurs poursuivants. Cette image de la fuite à travers bois est une figure narrative assez classique que le cinéma emprunte probablement aux contes et à la littérature² ${ }^{2}$.

5 Sur les six plans de la scène finale ${ }^{3}$, trois sont tournés en forêt. Les arbres y jouent un rôle scénique de structuration de l'espace. Dans le premier de ces plans, les arbres déterminent le trajet que les personnages empruntent pour descendre vers la rivière et la traverser afin de retrouver leurs chevaux. Un cheval blanc est attaché à un tronc en forme de fourche. Le panoramique d'accompagnement s'arrête lorsque le bord droit du cadre rejoint le tronc ${ }^{4}$. L'arbre sert donc de point de repère et de ralliement pour les gangsters mais aussi de point d'appui pour la représentation puisqu'il referme le cadre sur un espace où les personnages n'ont plus à retourner. Dans ce seul plan, les arbres ont donc une fonction scénique (orchestration spatiale des mouvements), narrative (ils témoignent de l'organisation des voleurs) et plastique (ils participent du dynamisme formel du champ). Les deux autres plans offrent une structure différente. Ce sont des plans fixes animés par le seul mouvement des cavaliers. Dans le premier, les quatre bandits sont poursuivis par les soldats dans une futaie. La course-poursuite se dirige droit vers le spectateur. Elle emprunte un chemin qui vient du fond du cadre et dessine deux courbes à travers les arbres. Les soldats abattent un des voleurs qui s'écroule au premier plan tandis que ses trois complices s'enfuient. Au plan suivant, dans une forêt à flanc de colline, les trois voleurs descendent de cheval et se regroupent pour partager leurs biens. Mais au fond du cadre, d'autres cavaliers arrivent à leur tour. Ils mettent pieds à terre et s'approchent en assaillant les bandits de coups de feu. Les arbres et les chevaux qui font écran entre les deux parties ne protègent pas les voleurs qui, un par un, sont abattus. Grâce à l'étalement des personnages dans le bois, le face à face est élargi à la pleine surface de l'image, surface soulignée par les arbres qui peuplent le champ de vision.

6 Ce film qui joue sur l'action, sur le montage et sur les changements d'angle, s'appuie en même temps sur une conception théâtrale de la scène encore empreinte de l'esthétique $d u$ « tableau ». L'espace s'y organise en effet en fonction du fond et du devant de l'espace scénique. Au point de vue presque systématiquement adopté, celui du quatrième mur de la tradition théâtrale, répond ainsi, en vis-à-vis, un mur de wagon ou de saloon qui ferme l'espace dans la majorité des plans. Néanmoins, dans le finale, ce fond de scène est remplacé par les arbres qui délimitent le champ visuel et forment un sous-bois dont l'intérêt est, semble-t-il, de créer des trouées, des passages, des chemins, des cachettes, des trajets pour les figures ici en action. La transition entre les scènes tournées en studio (wagon et saloon) et les scènes tournées en décor naturel (forêt) relève 
vraisemblablement de la volonté naissante du cinéma d'atteindre au réel. En effet, même si chaque plan de forêt correspond à un découpage plus théâtral que filmique (un plantableau équivaut ici à une scène : la fuite, la course-poursuite, l'échec), le tournage en sous-bois atteste du désir du cinéma narratif de placer la caméra sur le lieu de l'action et d'intégrer le réel à la narration. Dans cette séquence finale, la disposition des personnages parmi les troncs, leurs gestes et leurs mouvements ont probablement été prévus, sans doute ont-ils même été répétés dans le sous-bois comme ils l'auraient été dans un studio, pourtant la présence des troncs offre une représentation réaliste de cette poursuite. Si Porter s'appuie encore sur le modèle de l'espace scénique à travers le principe de la scène à trois côtés et le découpage des actions en tableau, le sous-bois lui permet d'assouplir ces repères culturels sans les supprimer totalement. Le cadrage de ces trois plans offre des vues différentes de la forêt. Or, ces changements de décors sont homogénéisés par les arbres, cadrés toujours à distance égale et qui participent ainsi du réalisme visuel du film. Outre leur fonction plastique et scénique, les arbres ont ici un rôle de garant d'un minimum de réalisme narratif.

7 E.S. Porter cadre ces sous-bois à hauteur de personnage. Ce cadrage réduit les arbres à de simples lignes verticales tout en les désignant dans leur totalité. Il laisse entrer dans ce récit la réalité de la forêt et avec elle la réalité de la nature. À l'instar de la figuration du corps, la figuration partielle de l'arbre suffit donc à en désigner le tout. Le cinéma d'animation, par exemple, peut en quelques minutes représenter l'arbre juste par la représentation d'un tronc sur lequel bute un personnage ou par celle d'une branche sur laquelle circule un chat ou un écureuil. Plus que tout autre genre de cinéma narratif, l'animation a fait de l'arbre une scène, un refuge, un habitat. Nous reviennent en mémoire des souvenirs d'arbres, légers et triviaux : les branches de chêne sur lesquelles des écureuils observent le monde et viennent se protéger des prédateurs; les branches qui servent de refuge et de tremplin à Félix le chat; les énormes troncs contre lesquels des dizaines de véhicules et de personnages viennent s'écraser à la fin d'une coursepoursuite. Comme Francis Hallé le souligne avec humour, « un arbre est un végétal tel que si vous rentrez dedans avec votre voiture, cette dernière sera cassée et vous devrez rentrer à pieds $»^{5}$. Comme le gros plan de visage ou de la main me dit le corps entier, le gros plan d'une branche ou d'un tronc me suggère l'arbre dans sa totalité. Même coupés par le cadre, la branche et le tronc, que mon imaginaire rassemblent, paraissent en effet indissociables. Dans sa constitution et par sa place même, le tronc est solidaire de chaque partie de l'arbre qu'il unifie. Selon le même principe, un plan de sous-bois désigne non seulement la totalité des arbres qui le constitue mais aussi la totalité organique de la nature à laquelle il appartient.

8 Au cinéma, la scène de sous-bois connaît de multiples variantes. Espace de la traque, de la course-poursuite comme on l'a vu précédemment, lieu de la perdition, lieu du passage d'un état à l'autre (de l'espace professionnel à l'espace public dans Le secret de Veronika Voss, R.W.Fassbinder), lieu de la promenade amoureuse (L'Enfance d'Ivan, Tarkovski) ou de la libération (Last days, Gus Van Sant ou Saraband, I.Bergman). Quelle que soit l'action qui s'y déroule, les arbres y sont traités avec égalité. Le remplissage du cadre par les troncs amoindrit la présence humaine; ce sont les arbres qui habitent l'écran tout en offrant à l'affect éprouvé par le personnage (peur, angoisse, liberté...) un territoire d'expansion.

9 Le rôle des arbres dans le cinéma de Tarkovski a déjà été beaucoup commenté. En effet, rares sont les œuvres ainsi maintenues entre deux plans d'arbres (celui qui ouvre L'enfance d'Ivan et celui qui clôt Le Sacrifice, son premier et son dernier film, donnant à 
l'arbre une dimension prophétique) et où la nature à un rôle si vif. La scène de sous-bois de L'enfance d'Ivan exploite en un peu plus de six minutes toutes les tensions d'une relation amoureuse faisant passer très rapidement les personnages d'un rapport de badinage à une relation de force et de soumission. L'arbre n'est pas le sujet premier de l'image, mais il joue cependant un rôle visuel décisif. Le sous-bois instaure une unification de la représentation; le regard ne se laisse attirer par aucun détail, frappé par la répétition du motif vertical du tronc et par l'absence de perspective; la perception de l'ensemble est globale. Seul ressortent les visages et les trajets des personnages mis en valeur par la blancheur des écorces et la composition des plans.

Cette scène offre un moment de détente dans un film de guerre. Le lieutenant Gatslev est amoureux d'une infirmière qui s'occupe du service sanitaire du camp. Par jeu, le capitaine Léonide Kholine essaie de la lui ravir. La scène se passe dans un bois de bouleaux, à la lisière duquel s'organise le départ des troupes pour le front. La scène du sous-bois est introduite par un panoramique de transition très rapide et bref sur la forêt vide. Ce plan monté en accéléré tend à déréaliser la scène qu'il introduit. Dans cet immense bois de bouleaux blancs, Kholine tente de séduire Macha, l'infirmière. La scène se divise en plusieurs momentsau cours desquels :

1. Kholine et Macha font connaissance

2. le jeune Gatslev part à leur recherche

3. Macha marche en équilibre sur un tronc d'arbre abattu

4. Kholine profite du prétexte de l'aider à enjamber un fossé pour la retenir dans ses bras et l'embrasser

5. le couple se sépare

6. Gatslev rejoint Kholine seul

Le découpage s'appuie sur un travail de cadrage et d'orchestration très rigoureux des mouvements de caméra. Le premier plan débute en plan rapproché sur le visage de Macha adossée à un tronc. L'arbre et Macha partagent le cadre. Aux questions posées hors champ par Kholine, elle répond en baissant les yeux avant de se retourner vers la gauche pour disparaître derrière l'arbre et s'éloigner. La caméra amorce alors un lent travelling sur le sous-bois ; le regard rencontre quatre ou cinq troncs avant de retrouver Macha qui a posé ses mains contre un autre bouleau. Kholine la rejoint. Ils poursuivent leur dialogue en marchant puis s'immobilisent dans un plan rapproché à deux avant de reprendre leur va-et-vient. Alors que le travelling se poursuit d'arbres en arbres, ils s'adossent contre les troncs, jouent dans ce labyrinthe ouvert qui offre des points de relais à leur trajet. Un bouleau s'interpose parfois entre eux, offrant à Macha une protection ou à Kholine un appui. Toute la scène est cadrée à hauteur de visage et le ciel n'apparaît jamais. Lorsqu'un travelling qui suit la courbe du terrain descend dans un fossé pour cadrer en contreplongée Kholine qui enjambe le fossé et retient Macha dans ses bras au-dessus du vide, la profusion des arbres cache toujours l'horizon. Après un jeu pervers qui consiste à attirer la jeune femme avant de lui ordonner de partir, Kholine s'éloigne dans la forêt. Un soupir de Macha révèle un cadrage subjectif sur la forêt; la caméra se lance dans une course désespérée entre les arbres qui se termine sur un tronc en gros plan. Dans un plan très bref, Macha de profil semble se précipiter la tête la première contre un arbre. Au plan suivant et dans le mouvement inverse, Gatslev arrive d'un pas rapide. Toute la scène résonne ici du sentiment de rivalité qui oppose et associe les deux camarades de guerre, Kholine et Gatslev. 
12 Les arbres accompagnent la chorégraphie de la scène. Mouvements de personnages et trajet de la caméra s'organisent autour d'eux, avec eux. Pourtant leur rôle dépasse la simple fonction scénique. La discontinuité spatiale créée par la présence répétitive des troncs participe de l'expression du malaise dans la relation des personnages. Les jeux de séduction et de possession, l'attente et la déception réciproques, la violence et la légèreté sont amplifiés par les rapports de lignes, les alternances de plein et de vide et l'étouffement visuel de cette plaine boisée à l'infini. D'autre part, lorsque le cadre est partagé par un tronc et un visage en plan rapproché, ou lorsqu'un tronc sépare deux personnages qui se font face, l'arbre devient le contemporain de la figure humaine. Il introduit dans l'image une tout autre dimension temporelle. Si l'arbre partage avec le visage la temporalité du cadre filmique, il figure simultanément une durée d'un autre ordre $^{6}$. Même cadré en gros plan, je perçois l'arbre comme une globalité, or cette totalité reste toutefois paradoxale, fixe et en expansion, immobile et pourtant animée, stable et cependant en évolution, toujours au même endroit et toujours différente. Dans la permanence de son immobilité, l'arbre introduit un rythme non humain, un interminable écoulement de durée, une profondeur de temps. L'écorce est la texture qui renferme en elle cette durée cachée. Détail agrandi de la forêt, elle porte ainsi à la surface mouvante de l'écran la promesse de ce qui dure. Notre perception du monde et du temps tourné vers l'avenir et les changements, orientée dans l'espoir d'un progrès, l'attente d'une amélioration, se heurte à l'arbre comme à l'image d'un trajet immobile.

13 C'est en jouant de la coprésence du visage et du tronc, que Tarkovski, dans cette scène de L'Enfance d'Ivan, inscrit ses personnages dans une durée qui dépasse le temps diégétique. Se glisse ici un dehors de l'image (non un ailleurs), car, entre le visage et le tronc s'inscrit non seulement un rythme temporel qui serait celui de la Nature mais, affleure également le temps de l'Histoire et celui de la mémoire du personnage. Malgré le décalage rythmique qui sépare le tronc et le visage, tous deux partagent des qualités de permanence et de fragilité. À l'égal de l'humain, l'arbre est aussi la présence de ce qui va disparaître. Il a la fragilité de ce qui est susceptible d'être abattu, arraché, taillé, brûlé (les arbres abattus comme des hommes dans le cinéma de guerre). Associer le visage et le tronc instaure, entre le rythme naturel et le rythme intérieur, un lien que le récit ne pourrait contenir par lui-même. Si pour Tarkovski le cinéma est l'art de «sculpter le temps ", la présence des arbres dans son œuvre donne une profondeur supplémentaire à son matériau filmique. Dans cette scène, le tronc confirme donc moins le réalisme de l'ensemble qu'il n'ouvre l'image à ce qui échappe à la narration.

L'arbre s'éloigne ainsi de plus en plus d'une simple fonction de décor pour guider le spectateur du réel vers quelque chose qui se montre et qui pourtant nous échappe, une dimension du visible où l'inexprimable se décèle. C'est aussi par le rapprochement visuel entre l'arbre et le visage que le plasticien italien Giuseppe Penone évoque parfois la cohabitation, le décalage et la complexité des rapports du temps humain et du temps botanique. L'intérêt de Penone pour l'arbre se traduit dans des propositions de dispositifs et d'installations très divers. S'il sculpte le bois, ce n'est pas pour en tirer la matière d'une abstraction ou d'une figuration quelconque mais pour, par exemple, extraire d'une poutre de charpente l'arbre qu'elle cache. La poutre est creusée jusqu'à son anneau de croissance et évidée de façon à dégager les nœuds à partir desquels les branches se sont formées. Les extrémités carrées et une face lisse de la poutre sont conservées pour souligner la coexistence du passé de l'arbre et de son présent utilitaire. Penone intervient parfois sur le bois vivant pour modifier son évolution, tresser des branches ou y introduire un corps 
étranger (la photographie d'un regard en gros plan par exemple). Une série d'œuvres en cristal nous intéresse particulièrement. Ce sont des sculptures transparentes qui ont la forme de bouts de bois, de bûches, de tronçons d'arbres de taille moyenne. Une double idée motive ces œuvres. D'une part, la notion de découpe y est fondamentale. L'arbre n'est pas représenté en entier comme c'est le cas dans d'autres œuvres de Penone, mais l'artiste nous en propose un fragment, un morceau. La seconde idée-force de ces pièces est la transposition du bois dans la transparence du cristal. Alors que l'écorce renvoie la lumière du soleil, le cristal est traversé par elle. Il est particulièrement difficile de fixer son regard à la surface de ces sculptures. Je perçois la branche et pourtant mon regard se poursuit vers le mur où elle est appuyée. Le regard perçoit le bout de bois, reconnait la texture de l'écorce mais il traverse la matière qui le constitue. Entraîné par le trajet de la lumière, il parcourt inconsciemment la durée suspendue dans cette matière fragile. Durée de vie de la branche interrompue par la scie qui l'a taillée, durée saisie dans l'éternité du cristal et à laquelle Penone me contraint à faire face. Il ne s'agit pas d'une expérience de la durée comparable à celle que le cinéma propose au spectateur, mais d'une véritable démonstration sculptée de la temporalité.

Fragmenté par le cadre de la représentation filmique ou interrompu par la main du sculpteur, le tronc nous offre ainsi une structure beaucoup plus complexe que l'apparente pauvreté plastique de sa forme. "Quelques précisions s'imposent, concernant le tronc d'arbre, écrit Francis Hallé. Existe-t-il un objet plus familier et en même temps plus énigmatique ? Il ressemble à une colonne - comme celles de la maison carrée à Nîmes... » ${ }^{7}$. Pour notre regard, formé par l'artialisation in visu, le tronc effectivement ressemble à une colonne. Dans l'installation d'Abbas Kiarostami intitulée Forêt sans Feuilles, l'arbre et la colonne se confondent parfaitement. Initialement conçue pour une exposition sur les jardins persans au Musée d'art Contemporain de Téhéran en septembre 2004, cette installation recrée en trois dimensions un sous-bois artificiel ${ }^{8}$. Les arbres sont figurés par d'immenses tubes recouverts de photographies de troncs d'arbres. Il s'agit littéralement d'une forêt de colonnes, d'un "jardin sans feuilles " comme celui que décrit le poète Mehdi Akhavan Saless dans un poème que Kiarostami a mis en exergue de l'installation'. Pour réaliser chaque arbre, chaque colonne, quatre-vingt photographies ont été prises et assemblées numériquement ${ }^{10}$. Ces tirages recouvrent des tubes de diamètres variables qui, disposés dans l'espace, reconstituent une forêt d'intérieur. Un plafond lumineux crée une lumière continue et sans ombre tandis que les murs de l'installation offrent une surface réfléchissante qui démultiplie les arbres de toutes parts, amplifiant ainsi les dimensions de cette forêt.

Dans l'œuvre d'Abbas Kiarostami, plusieurs films antérieurs à l'installation de La Forêt sans feuilles comportent des scènes de sous-bois. Les scènes tournées en forêt dans Où est la maison de mon ami ? (1987), Et la vie continue (1991) et Au travers des oliviers (1994), sont cadrées, à hauteur de regard, au niveau des troncs d'oliviers. Les feuillages ou le sol sont déjà exclus de l'image dans une composition qui élimine le ciel. Les troncs dessinent d'épaisses lignes verticales qui traversent le cadre et parfois s'entrecroisent. Ces lignes sont, sans hésitation, coupées par les bords du cadre. Les feuillages entrent rarement dans ces plans. La coupure du bas et du haut des arbres donne davantage de latéralité à une image fermée dont la profondeur de champ est réduite. Fermeture du cadre, cloisonnement spatial, remplissage du cadre par les troncs, tous ces éléments répondent $\mathrm{du}$ principe de saturation du champ dans sa largeur. Le remplissage de l'espace par des troncs évoque la structure d'une miniature persane du XIVème siècle qui appartient à 
L'Histoire universelle de Rachîd ed-Dîn : "L'arbre sacré de Bouddha " ${ }^{11}$. Cette peinture représente cinq troncs d'arbres disposés asymétriquement dans toute la largeur d'une page. Comme les films évoqués ci-dessus, cette miniature ne s'intéresse qu'aux troncs. Deux arbres très massifs et noueux, ce sont peut-être des chênes, entourent trois troncs plus frêles dont le contour bien marqué en noir se détache sur un fond de paysage plus abstrait. Les troncs n'ont rien de rigide, ils poussent dans des directions opposées et sont animés par des variations de couleurs, terre et gris bleu. La caractéristique majeure de cette miniature est un cadre tranchant qui interrompt brutalement la représentation des arbres. Selon Basil Gray, le fait que «le cadre coupe toujours la composition, sans compromis $»^{12}$, est une constante due à une nécessité d'équilibrer ici, texte et image dans la mise en page. Ce modèle visuel se confond avec celui des plans de sous-bois du cinéma de Kiarostami bien que le cinéaste se défende de toute influence ${ }^{13}$.

Alain Bergala souligne que ce type de plans est celui de la communication bloquée. Il leur donne le nom de plans «code barre». "C'est, dit-il, le plan par excellence, chez Kiarostami, de la difficulté du personnage à avancer, mais encore plus de communiquer avec l'autre, comme si ces barres verticales (le plus souvent des troncs d'arbres) empêchait la parole d'être entendue ou comprise par l'autre " ${ }^{14}$. Dans Le Vent nous emportera (1999), Behzad, le personnage principal dialogue avec l'enfant dans un sous-bois de bouleaux. La scène est ici plus proche de celle de L'Enfance d'Ivan ${ }^{15}$ que de celle de la miniature du Djami' et-Tawârikh. Cependant le dialogue entre les personnages y est tout aussi difficile. Alors que le garçon donne à l'étranger des nouvelles de la vieille dame malade, ce dernier découvre que le souhait du garçon serait qu'elle guérisse alors qu'il souhaite au contraire que la mort vienne au plus tôt.

Dans la Forêt sans feuilles, Kiarostami vide ce que serait l'espace scénique de ses personnages et les remplace par des spectateurs virtuels. Il nous permet ainsi d'entrer dans l'espace de la représentation. Dominique Païni constate qu'« en termes d'action, les visiteurs sont encouragés à devenir, dans La Forêt sans feuilles, les personnages des films de Kiarostami, même si cette transformation est plus plastique que narrative $»^{16}$. Des trajets visuels mais aussi physiques se mettent ainsi spontanément en place. Le regard n'a pas à parcourir la surface de la représentation, mais le corps entier est invité à se promener dans ce paysage reconstitué. Alain Bergala a relevé le côté ludique de cette installation ${ }^{17}$. Effectivement le spectateur prend plaisir à circuler entre ces arbres. On peut s'y promener, y découvrir des inscriptions sur les troncs (les photographies laissent apparaitre des traces des signatures, des initiales, des prénoms de garçons et de filles accompagnés d'une date), y jouer à cache-cache, y photographier des inconnus, poser comme des touristes dans une vraie forêt.

Mais ce dispositif ludique est aussi une démonstration figurale du rapport du spectateur à l'œuvre. En fait l'installation place directement le spectateur dans la fiction que Diderot avait imaginée comme méthode de description des peintures de paysages. "C'est une assez bonne méthode, pour décrire des tableaux surtout champêtres, que d'entrer sur le lieu de la scène d'abord par le côté droit ou le côté gauche, et s'avançant sur la bordure d'en bas, de décrire les objets à mesure qu'ils se présentent $»^{18}$. La méthode descriptive de Diderot consiste en un parcours ou un cheminement du regard dans l'œuvre qui conduit le spectateur à entrer littéralement dans l'espace de la représentation, dans la scène. Le critique devient un promeneur dans le tableau, un promeneur qui écrit simultanément ce qu'il voit. Ainsi peut-on lire chez Diderot de véritables trajets: "Je m'établis sur la bordure et je vais de la droite vers la gauche. Ce sont d'abord de grands rochers, j'aperçois 
un paysan assis et un peu au-dessous de ce paysan, une paysanne assise aussi (...) ils semblent écouter, et ils écoutent en effet un jeune musicien qui joue (...) d'une espèce de mandoline. Je continue mon chemin ; je quitte à regret le musicien parce que j'aime la musique et que celui-ci a un air d'enthousiasme qui attache (...). Si j'allais plus loin j'entrerais dans un bocage, mais je suis arrêté par une large mare d'eaux qui me font sortir de la toile $»^{19}$. Diderot décrit ici un tableau du peintre Le Prince, intitulé Le Musicien champêtre. C'est de l'intérieur du tableau que le promeneur voit les objets qu'il rencontre, de l'intérieur du paysage que le critique écrit sur sa représentation. Si l'œuvre aménage des chemins pour le regard (on pense inévitablement à Paul Klee), le principe d'une installation est d'aménager un espace, des trajets, des sensations pour le corps du spectateur. Ici, la description pourrait commencer par n'importe quel tronc. L'installation de la Forêt sans feuilles témoigne d'une condensation de l'expérience esthétique et physique de la forêt. Ces tubes verticaux n'ont rien à voir avec des arbres, j'y perçois pourtant une expérience concentrée de la forêt. Je peux non seulement y tracer mes propres chemins, mais j'y retrouve aussi de multiples expériences esthétiques du sous-bois qui complètent ma perception sensible de l'artifice. La Forêt sans feuilles déploie et agrandit un palimpseste mental de plans de forêt filmiques et de tableaux de forêt que je suis maintenant invitée à parcourir physiquement. La Forêt sans feuilles peut se lire comme un morcellement de la perception, une forêt dont on ne perçoit que les troncs, pas de branches, pas de ramure, de feuilles, ni de racines; un fragment de forêt, condensé par des expériences visuelles préalables. Cependant l'installation n'est pas qu'un fragment de forêt. La surface réfléchissante qui entoure le sous-bois reflète un monde mouvant et instable. Les arbres y perdent la netteté de leurs contours et les visiteurs leur propre ressemblance. Comment ne pas penser ici au palais des quarante colonnes d'Ispahan où vingt colonnes de pierre se reflètent à la surface de l'eau dans les bassins aménagés à cet effet ? Elles se dédoublent ainsi en quarante colonnes où se perd la mesure de la réalité. Les vingt colonnes d'Ispahan se dédoublent à la verticale, une seule fois chacune; les troncs de la Forêt sans feuilles se démultiplient à l'horizontale dans des reflets sans fin. L'installation est à la fois une dilatation spatiale de représentations de sous-bois (picturales et cinématographiques) et un dispositif qui condense en un fragment perceptif notre expérience de la nature et de l'architecture. Le dispositif de Kiarostami déploie ainsi dans l'espace l'action psychique de l'artialisation du regard. En plaçant le spectateur dans cette forêt de colonnes tapissées comme des arbres, il lui renvoie le processus mental par lequel son regard esthétique se forme.

21 Il est difficile désormais de traverser un sous-bois et de voir les arbres autrement que par le tronc. Le regard effectue de lui-même un travail de condensation par fragmentation du tout. Il rassemble la complexité de la structure et de la vie de l'arbre sur un élément qui a la simplicité d'une colonne, mais qui ne se réduit pas pour autant à un détail schématique. Or paradoxalement, lorsque le regard se concentre sur le tronc, ce dernier provoque aussi une dilatation temporelle de la durée. L'écorce qui partage le même présent que nous, désigne en même temps le lent processus temporel qui anime la vie de l'arbre et c'est finalement au même rythme que strate par strate, l'artialisation opère. Si un plasticien comme Giuseppe Penone joue du bois et du cristal pour construire ses démonstrations théoriques, le jeu d'Abbas Kiarostami est de passer par un matériau autre que le bois pour nous dire que la nature s'infiltre en nous, travaille nos modes de pensée et que la « mémoire de l'arbre » nous imprègne au-delà de l'imaginable. 


\section{NOTES}

1. Comme le souligne Daniel Serceau, l'arbre est «simple décor, motif plastique, objet symbolique ", L'arbre dans le paysage, dir. Jean Mottet, éd. Champ Vallon, p. 99. Dans cet ouvrage, Jean Mottet a formulé très clairement la problématique de l'analyse de l'arbre dans la représentation: «l'étude du motif de l'arbre relève-t-elle de l'analyse des œuvres dans leur constitution formelle ou de l'étude iconologique qui privilégie le motif dans sa relation à un espace plus large? », op. cit., p. 7.

2. Je pense à la littérature fantastique ou aux récits de chasse, aux contes nordiques. Dans les contes de Grimm, la forêt est, par exemple, un lieu où les personnages « courent les risques de se faire détrousser, assassiner, dévorer, métamorphoser ». Simon Schama, Le paysage et la mémoire, éd. du Seuil, 1999, p. 125.

3. Nous utilisons le terme de "plan " par commodité car, rappelons le, à cette époque, le récit cinématographique s'écrivait encore en "tableaux». Sur le plan syntaxique, ce film marque néanmoins un tournant ainsi que le suggère le célèbre plan rapproché d'un des bandits tirant en direction du spectateur et dont le catalogue Edison préconisait de le placer soit au début, soit à la fin du film. À la faveur de cet élément, germait ainsi l'idée, certes encore assez vague, du plan au sens de fragment narratif qui rompait avec la temporalité et l'espace stricts du « tableau ».

4. Ce mouvement de caméra qui ouvre partiellement l'espace scénique et visuel nous éloigne déjà de l'esthétique du «plan-tableau ».

5. Francis Hallé, Plaidoyer pour l'arbre, éd. Actes Sud, 2005, p. 22.

6. «Le tronc d'arbre est en réalité une structure complexe, stratifiée, concentrique, et qui ne peut être comprise que si l'on prend en compte les effets de l'écoulement du temps ». Francis Hallé, op.cit., p. 61

7. Francis Hallé, ibid., p. 61.

8. Présentée au Victoria \& Albert Museum à Londres en avril 2005 et à Barcelone au printemps 2006.

9. Le poème de Saless date de 1965 (Saless est surnommé Omid, c'est-à-dire Espoir), Kiarostami l'avait déjà mis en exergue d'un court récit intitulé «Le Passager » : "Mon jardin, / Il serre son ciel dans ses bras,/ le nuage, dans sa fourrure humide et froide./ Le jardin sans feuilles,/ est seul jour et nuit,/ avec son silence pur et triste.// La pluie est son instrument, le vent et son chant./ Son habit est une soutane dénudée./ Il lui faut un autre habit,/ d'une flamme dorée, le vent a tissé son corps. // Que tout pousse ou ne pousse pas, là ou il veut/ et n'importe ou,/ ni jardinier, ni passant,/ le jardin des sans espoir,/ n'attend aucun printemps. // Si de ses yeux ne se réfléchit aucun rayon de chaleur,/ et sur sa face ne pousse aucune feuille de sourire,/qui dit qu'un jardin sans feuille n'est pas beau ?/ Il raconte à la terre l'histoire des fruits élevés au ciel/ ( et couchés maintenant dans le cercueil)// Le jardin sans feuilles/ son sourire un sang larmoyant./ Là, sur son cheval à crinière jaune trotte éternellement/ le roi des saisons, l'automne. "Trafic $\mathrm{n}^{\circ} 2$, printemps 1992, pp. 71-73.

10. Quatre appareils photos placés en cercle sur des axes verticaux autour de l'arbre permettent de déclencher quatre prises de vues tous les 20 centimètres de façon à photographier la presque totalité du tronc. Les photos numériques sont ensuite assemblées par ordinateur et tirées en très grand format.

11. Djami et-tawârikh (Histoire universelle) de Rachîd ed-Din, Rab-i-Rashidi, 1314 (H. 10 cm ; L. 25 $\mathrm{cm}$ ), Londres, Royal Asiatic Society. Basil Gray, La peinture persane, éd. Skira, 1995.

12. Basil Gray, La peinture persane, op.cit., p. 26. 
13. Le cinéaste se dit surpris lorsqu'on lui montre les ressemblances qui existent entre certaines miniatures et des plans de ses films. Il reconnaît a posteriori l'existence de ce lien, mais il ne s'agit pour lui ni d'une source d'inspiration, ni même d'un rapport d'influence. Lorsque j'ai eu la possibilité de lui montrer ces miniatures mises en parallèle avec des plans de ses films, il les a regardé avec intérêt mais ne les a pas commentées. Ce n'est que dans son entretien avec Jean-Luc NANCY qu'il semble en avoir parlé : « Une étudiante en thèse m'a montré une série d'images dans lesquelles j'ai vu une ressemblance surprenante entre certains plans de mes films et quelques détails de miniatures : la présence d'arbres et de chemins sinueux. Pourtant, je ne me suis jamais vraiment senti proche de la miniature persane. C'était pour moi une découverte étrange de voir qu'il y avait un rapprochement possible. Peut-être parce que nous vivons les miniaturistes et moi, dans la même nature et dans le même pays, sommes nous arrivés à une même pensée autour de l'arbre ou des chemins en zigzag ? Il ne s'agit pas de copies volontaires mais probablement de véritables ressemblances. » L'Évidence du film, Yves Gaevert Éditeur, 2001, p. 81.

14. Alain Bergala, Abbas Kiarostami, Petits Cahiers, Cahiers du cinéma, 2004, p. 78

15. Le jeune garçon traverse le ruisseau en empruntant un tronc d'arbre en guise de pont pour rejoindre son ami, on retrouve ainsi le geste de Macha en équilibre filmée par Tarkosvki.

16. Dominique Païni, « The present exposed, \#Notes

on the Installations of Abbas Kiarostami » in Erice Kiarostami Correspondences, Centre de cultura contemporània de Barcelona, 2006, pp. 130-131 (t.d.a.).

17. Cahiers du cinéma, février 2006, p.88

18. Diderot, Euvres complètes, tome 7, éd. Chronologique, Club français du livre, 1970, pp. 146-147.

19. Ibid, Tome 7, p. 244.

\section{AUTEUR}

\section{CAROLINE RENARD}

Docteur en Etudes Cinématographique

Chargée de cours, Université de Provence 